\title{
Exploration on Function Reconstruction and Evaluation of Community TCM Service Based on the Perspective of Health Service
}

\author{
Chao Wang \\ School of Physical Education, Shaanxi Normal University, Xi'an Shaanxi, 710119, China
}

Key words: Health service; Community, TCM, Function reconstruction, Evaluation

\begin{abstract}
In recent years, the concept of health service has been proposed and applied in all sectors, so the TCM (traditional Chinese medicine) service carried out in community should also advance with times and adjust its own job functions under the guidance of this concept. To better understand the implementation of functions, it is also required to refine the evaluation work. This paper will study the problems of functional adjustment and assessment.
\end{abstract}

\section{Introduction}

A community, which is the most basic unit in people's life, plays an important role in inspecting and managing the residents' physical health and quality. As a traditional way of health guide, TCM occupies an important position in Chinese medical and health filed and plays a significant role in the residents' health care. Thus, this paper mainly explores and analyzes the function of TCM service in the community agency in the hope of accelerating the further development of health service industry of China.

\section{Proposal and practice of the concept of health service}

\section{Proposal of the concept of health service}

In essence, the basic concept of health service differs from the medical service and health service. According to the analysis on current situation, this concept was proposed by WHO (World Health Organization) in the middle of 1980s at the earliest, and was further perfected and developed in the process of continuous social development. The brand-new concept of health service is a perfect and comprehensive concept of health service established from physiology, psychology, social adaptability, individual moral accomplishment and other aspects.

In October 2013, Some Opinions on Promoting the Development of Health Service Industry issued by the State Council of China further perfected the concept of health service and also pointed out that the objective of perfecting the health service system by the state is, in essence, to enhance people's health level and psychological health level. Therefore, the overall work system of health service includes medical service work, health management work, health promotion work, health insurance work and all other relevant work concerned. As a result, after the issuance of Opinions, compared with before, the health service level in China was significantly improved and, meanwhile, drove the rapid development of many industries and trades. For instance, the healthy food, health equipment, health products, medicines were managed in a more standardized way.

Medical service and hygiene service

Reasonably handling the relationship between medical service and hygiene service is very crucial for improving the overall level of health service. Generally speaking, there is an obvious correlation between medical service and hygiene service. However, to accelerate the development of health service, be sure not to mix them up. At first, the medical service refers to that a medical institution 
offers disease prevention, disease diagnosis, disease treatment, publicity and education of disease and health knowledge and other series of activities to the patients by using the internal personnel, medicines, apparatuses and other resources in the institution. However, such medical service is usually offered in a specific place so it has an obvious regional limitation.

The hygiene service usually means that the hygiene department provides community residents with certain hygiene service for a purpose. In this process, specific medical service resources such as human resource, fund, technology and information are also needed. Compared with the medical service, the hygiene service is relatively limited at the technological level but is more flexible and convenient in the forms of service.

It is not difficult to find from analysis on them that, compared with medical service and hygiene service, the service of health service has the more generalized value of medical service, so all-around progress and development will be made in the concept of health service.

\section{Function reconstruction of TCM service in community based on the perspective of health service}

\section{Establish reasonable development goals}

As for the TCM service based on the perspective of health service, to fully realize the goals and development potential, it is required to reasonably plan the overall development goals. Firstly, unilaterally, the basic goal of health service is to improve the Chinese community residents' health concept and health level so as to meet residents' most fundamental requirement for health service; the developmental goal of health service is to enhance residents' health awareness and to further accelerate the development of health industry; the final goal of health service is to continuously improve the overall health level of Chinese people, to achieve the reasonable and optimized development of our industry on this basis and to guarantee the healthy and steady development of national economic structure. Secondly, our country has two goals for the development of TCM service. On the one side, use the traditional TCM knowledge to give reasonable guidance for health knowledge to residents; on the other side, stimulate the development, propagation and promotion of TCM culture when promoting the improvement of residents' health level. Thus it can be seen that both health service and TCM service consider improving residents' health level as the basic requirement and goal.

\section{Clearly define the problems to be solved}

According to the analysis, it is clear that the entire system function of community hygiene service is in urgent need of perfection with the ceaseless improvement of residents' living standard. Especially, the functions and levels of medical service, health care and maternity service need improving and developing. Within this stage, health service improves people's health awareness, simultaneously makes requests of a higher level for the temperature rise service in community and promotes the change of modernized community hygiene service. Namely, within the stage, community hygiene service shall be changed from offering health treatment to residence into offering disease prevention and health care to residents. Thus, how to effectively complete the mutual coordination of TCM function and health service function within this stage has become a critical problem which must be thought deeply by the health service administration department concerned.

\section{Functional requirements of TCM service in community}

To fully improve the overall level of community health service and realize the change of fundamental function of TCM, It is needed to further reasonably plan and study the fundamental functions of TCM service and adopt supervision and administration and other forms to practically ensure such functions to be truly realized in community service agencies.

The fundamental functions of community TCM service mainly include the follow aspects: firstly, TCM service must adhere to the people-oriented principle, do everything from people's practical interests, fully respect residents' health willingness and provide reasonable and effective TCM service on this basis; secondly, gradually change from TCM treatment into the full-course management of disease prevent and treatment to enhance residents' satisfaction and well-being; 
thirdly, further expand the publicity so as to further improve residents' awareness of TCM health care.

\section{Evaluation on community TCM serviceability based on the perspective of health service}

\section{Design of evaluation system}

Based on the analysis on present situation, the TCM serviceability needs to be perfected. Especially, there are many problems in the propulsion of community service such as imperfect service function, difficulty in quality guarantee and insufficient serviceability. It is badly in need of finding a practical and effective way to carry forward the TCM service to have basis; in addition, maximally guarantee the quality to thus enhance the TCM serviceability, promote it to overcome all obstacles during propulsion in communities and to get rid of realistic difficulties. In recent years, the concept of heath service has been energetically popularized. It is of great significance to put forward such a concept, which gave a guiding light for TCM service to some extent and plays a critical role in the evaluation of serviceability. Building a grading system is the important driving force to improve TCM serviceability. The key part of evaluation system is the corresponding relevant indicator, which is the core content in the evaluation system and guarantees the realization of value goal in a sense. The value goal is the most important part in the system, is the basis for the content of grading indicator, shows the value standard in the form of internalization to some extent and effectively monitors the assessment process.

The effective application of community TCM can have an unexceptionable effect. In accordance with its service function, social hygiene service, the "six-in-one" requirement and the overall development direction of health service, the evaluation can be carried out from three dimensions. The first dimension is the fundamental construction indicator; the second dimension is the service evaluation indicator; the third dimension is the effect evaluation indicator. In this way, boost the healthy construction of community service and build a related evaluation system. Infrastructure, which is the basic condition for the development of health service, is the prerequisite for TCM to play its role. Besides, the level of infrastructure reflects back the degree of importance of health care to some extent and is also an embodiment of the TCM function. With respect to the conducting of health service, it is a process indicator in essence and shows relevant contents of health service. It is needed to select a process indicator from many levels such as medical rehabilitation, prevention, health care and health management. To adapt to the above-mentioned functions, compared with the previous assessment standards, there are changes in the following two indicators which are respectively prevention and health care as well as health management. The two aspects become more rich and specific in their contents. As for health promotion, it is a result indicator in essence, can visually and three-dimensionally show the effect of health service, is consistent with the local people's need, and complies with their goal of health development. Content expansion is needed in the indicators related to health promotion. Apart from the existing satisfaction indicator, health improvement indicator should also be added so the essential effect of health promotion can be analyzed in the long run.

\section{Selection of evaluation indicator}

\section{Basic construction indicators}

Basic construction indicators, which are the basic conditions of community health service, also can show the investment in TCM service to some extent and covers two aspects: the policy background indicator and community building indicator. The policy background indicator is the important embodiment of the macro control by the government, the government's supervision strength for community health service and covers the aspects below: firstly, policies or documents concerning TCM service issued by the government; secondly, fund investment in TCM service. The community building indicator, which mainly reflects the construction level of community health service, chiefly includes three aspects, which are respectively department setting, equipment configuration and personnel allocation. The department setting mainly covers the TCM consulting room, TCM pharmacy and medicine decocting room; in addition, it also covers the recovery room, health care 
room etc. Equipment configuration: basic office equipment, TCM equipment matching such as acupuncture, massage and scraping therapy, and equipments for TCM pharmacy. Personnel allocation basically means the personnel engaged in community health service mainly including the TCM doctors, medicament allocation personnel, bed nurses etc.

Service evaluation indicator

Community health service has caught sufficient attention and personnel engaged in TCM are gradually growing. According to the analysis on current situation, the TCM service has a very explicit goal; namely, the TCM service is health-oriented. In accordance with the requirements of health service and the characteristics of TCM service, with reference to related documents, and based on the analysis and consideration at the project level, relevant indicators for building community health service mainly covers four aspects: prevention and health care, TCM treatment, TCM recovery and TCM health management. The prevention and health care includes many aspects such as health care via acupuncture, health care via massage, health care in four seasons and health care via working method, mainly investigates the development proportion of the project in communities and also needs to refer to the probability of personnel participation. The chief contents involved in TCM treatment are as follows: non-chronic diseases, chronic diseases, therapeutic methods, proportions, and development of community health service such as door-to-door service. Workers need to timely know the development of the above-mentioned activities in communities. The project indicator of TCM recovery includes the following contents: the specific implementation of dominant disease service, rehabilitation of disable patients, malignant tumor rehabilitation and other service content. The health management service can be elaborated from two aspects which are respectively health management and health education. As far as community health management is concerned, each community should formulate feasible TCM prevention measures and determine health care schemes based on its own practical situation or should study out a personalized service scheme in accordance with the specific requirements of community health service. Health education, which mainly helps the community people to know the health knowledge from the knowledge level, is mainly conducted in the form of giving a lecture, or by establishing a health consultation platform to discuss the development of community health education.

Effect evaluation and analysis indicator

TCM service has caused high concern in our country and has been effectively implemented in communities. According to the analysis from a certain level, the level of community health promotes the setting of relevant indictors in a way, provides a visual way to know the implementation effect of health service and is a real reflection of the improvement of community diseases. Generally, it includes two aspects, which are the health promotion indicator and service development indicator. As for the health promotion indicator, the community people are mainly used as the objects of study. Changes in their health behaviors and thoughts after TCM service can show the current situation of community health management. For specific indicators, refer to the following: development of health service, spreading rate of hygiene knowledge, formation probability of health behavior etc. With respect to the service development indicator, the community people's satisfaction or acceptability of health service can show the specific implementation of TCM service as well as the development foundation. The specific reference indicators are shown below: residents' satisfaction and acceptability, absorbing ration of health activities etc.

\section{Conclusion}

In general, that the improvement of people's living standard promotes their gradual improvement in the awareness of health service is mainly shown in the contents, forms, personnel and continuously lifting level of community health service. This paper discusses the current situation of community TCM service from many aspects, proposal of relevant concepts as well as the gradual implementation of indicators, and further accelerates the ceaseless optimization of community health service in our country so as to attain better effects. 


\section{Acknowledgements}

This paper is the project of Shanxi Federation of Social Science; project name: Study on Construction of Health Service System for Community Residents in Xi'an; No.: (2015Z004).

\section{References}

[1] Wang Gaoling; Feng Ya. Thought on Function Reconstruction and Evaluation of Community TCM Service-Perspective Based on Health Service. Journal of Nanjing University of Traditional Chinese Medicine (Social Science), 2014 (04).

[2] Wang Gaoling; Ji Jiao. Analysis on Construction and Improvement of Community TCM Team's Health Serviceability. Medicine and Society, 2015 (01).

[3] Cheng Wei. Economic Benefit Evaluation and Mode Study of Medical Service in Community Hygiene. Shandong Traditional Chinese Medicine University, 2012.

[4] Bian Li. Study on Evaluation Methods and Influence Factors of Community TCM Service. Hubei University of Chinese Medicine, 2012.

[5] Fan Boyuan. Study on the Demand and Supply of TCM in Community Hygiene Service. Chinese General Practice, 2011 (25). 